\title{
Fractionally spaced blind equalization with low-complexity concurrent constant modulus algorithm and soft decision- directed scheme
}

\author{
S. Chen ${ }^{1, * \dagger}$ and E. S. Chng ${ }^{2}$ \\ ${ }^{1}$ School of Electronics and Computer Science, University of Southampton, Highfield, Southampton SO17 1BJ, U.K. \\ ${ }^{2}$ School of Computer Engineering, Nanyang Technological University, Nanyang Avenue, Singapore 639798, Singapore
}

\section{SUMMARY}

The paper proposes a low-complexity concurrent constant modulus algorithm (CMA) and soft decisiondirected (SDD) scheme for fractionally spaced blind equalization of high-order quadrature amplitude modulation channels. We compare our proposed blind equalizer with the recently introduced state-of-art concurrent CMA and decision-directed (DD) scheme. The proposed CMA + SDD blind equalizer is shown to have simpler computational complexity per weight update, faster convergence speed, and slightly improved steady-state equalization performance, compared with the existing CMA + DD blind equalizer. Copyright (C) 2004 John Wiley \& Sons, Ltd.

KEY WORDS: fractionally spaced blind equalization; quadrature amplitude modulation; constant modulus algorithm; decision-directed adaptation

\section{INTRODUCTION}

Blind equalization improves system bandwidth efficiency by avoiding the use of a training sequence. Furthermore, for multi-point communication systems, training is infeasible and blind equalizer provides a practical means for combating the detrimental effects of channel intersymbol interference (ISI) in such systems. For communication systems employing high bandwidth-efficiency quadrature amplitude modulation (QAM) signalling [1], the constant modulus algorithm (CMA)-based equalizer is by far the most popular blind equalization scheme [2-5]. It has very simple computational requirements and readily meets the real-time computational constraint. The CMA is also very robust to imperfect carrier recovery. A particular problem of the CMA, however, is that it only achieves a moderate level of mean square error (MSE) after convergence, which may not be sufficiently low for the system to obtain adequate bit error rate (BER) performance. A possible solution is to switch to a decision-

\footnotetext{
*Correspondence to: S. Chen, School of Electronics and Computer Science, University of Southampton, Highfield, Southampton SO17 1BJ, U.K.

†E-mail: sqc@ecs.soton.ac.uk 
directed (DD) adaptation which should be able to minimize the residual CMA steady state MSE [6]. However, as pointed out in Reference [7], in order for such a transfer to be successful, the CMA steady state MSE should be sufficiently low. In practice, such a low level of MSE may not always be achievable by the CMA.

De Castro et al. [7] have suggested an interesting solution to this problem. Rather than switching to a DD adaptation after the CMA has converged, they have proposed to operate a DD equalizer concurrently with a CMA equalizer. To avoid error propagation due to incorrect decisions, the DD weight adaptation only takes place if the CMA adaptation is judged to have achieved a successful adjustment with high probability. At a cost of slightly more than doubling the complexity of the very simple CMA, this concurrent CMA + DD equalizer is reported to obtain a dramatical improvement in equalization performance over the CMA [7]. Among various low-complexity blind equalization schemes for high-order QAM channels (e.g. References [8-11]), this concurrent CMA + DD scheme represents a state-of-art technique. Another blind equalization scheme, which is relevant to the proposed concurrent CMA and soft decision-directed (SDD) blind equalizer, is the bootstrap maximum a posteriori probability (MAP) blind equalizer [12, 13].

The bootstrap MAP blind scheme was originally derived by Karaoguz and Ardalan [14] for the 4-QAM case and extended to $M$-QAM channels by Chen et al. $[12,13]$. It is only slightly more complex than the CMA in terms of computational requirements and it has been shown to outperform the CMA + DD scheme, in terms of convergence rate and steady-state performance $[15,16]$. A drawback of this bootstrap MAP scheme is that its adaptive process requires $L$-stage switchings, where $L=\log _{2}(M) / 2$, and each stage of adaptation needs a different set of algorithm parameters. Thus, tuning of the bootstrap MAP algorithm is quite complicated. The proposed CMA + SDD scheme may be viewed as operating a CMA equalizer and a last-stage bootstrap MAP equalizer concurrently, and it does not require complicated switching. The proposed CMA + SDD scheme has a simpler complexity than the CMA+DD scheme. Simulation results obtained under a fractionally spaced equalizer (FSE) setting show that the CMA + SDD algorithm has a faster convergence rate and slightly better steady-state performance, compared with the CMA + DD scheme.

\section{LOW-COMPLEXITY BLIND EQUALIZATION}

For notational simplicity, blind equalization with a $T_{\mathrm{s}} / 2$-spaced FSE is considered, where $T_{\mathrm{s}}$ denotes the symbol period. The baseband discrete-time model of communication system with a $T_{\mathrm{s}} / 2$-spaced FSE (e.g. Reference [17]) is depicted in Figure 1. For notational convenience, the

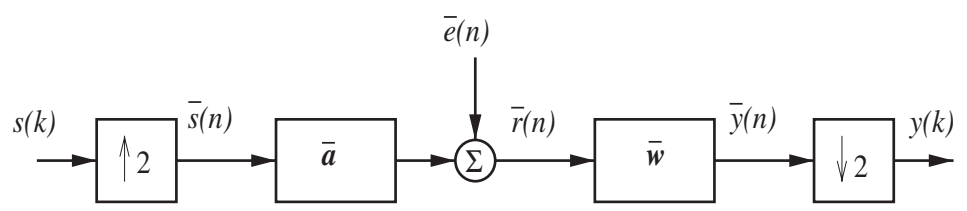

Figure 1. Multirate baseband model of communication system with $T_{\mathrm{s}} / 2$-spaced equalizer, where $T_{\mathrm{s}}$ denotes symbol period, the index $k$ indicates $T_{\mathrm{s}}$-spaced quantities and index $n$ indicates $T_{\mathrm{s}} / 2$-spaced quantities. 
index $k$ is reserved for $T_{\mathrm{s}}$-spaced quantities and index $n$ for $T_{\mathrm{s}} / 2$-spaced quantities throughout the discussion. The transmitted $T_{\mathrm{s}}$-spaced complex symbol sequence $s(k)=s_{R}(k)+j s_{I}(k)$ is assumed to be independently identically distributed (i.i.d.) and the symbol constellation is $M$ QAM with the set of all the symbol points defined by

$$
\mathscr{S}=\left\{s_{i l}=(2 i-Q-1)+j(2 l-Q-1), \quad 1 \leqslant i, l \leqslant Q\right\}
$$

where $Q=\sqrt{M}=2^{L}$, and $L$ is an integer. The received $T_{\mathrm{s}} / 2$-spaced signal sample is

$$
\bar{r}(n)=\sum_{i=0}^{2 N_{c}-1} \bar{a}_{i} \bar{s}(n-i)+\bar{e}(n)
$$

where the $T_{\mathrm{s}} / 2$-spaced sequence $\{\bar{s}(n)\}$ is a zero-filled version of the transmitted symbol sequence $\{s(k)\}$ defined by

$$
\bar{s}(n)= \begin{cases}s(n / 2) & \text { for even } n \\ 0 & \text { for odd } n\end{cases}
$$

the channel is specified by the $T_{\mathrm{s}} / 2$-spaced complex-valued channel impulse response (CIR) given by

$$
\overline{\mathbf{a}}=\left[\begin{array}{llllll}
\bar{a}_{0} & \bar{a}_{1} & \bar{a}_{2} & \bar{a}_{3} & \cdots & \bar{a}_{2 N_{c-1}}
\end{array}\right]^{\mathrm{T}}
$$

with $N_{c}$ corresponding to the $T_{\mathrm{s}}$-spaced CIR length, and the $T_{\mathrm{s}} / 2$-spaced sample $\bar{e}(n)=$ $\bar{e}_{R}(n)+j \bar{e}_{I}(n)$ is an i.i.d. complex Gaussian white noise with $E\left[\bar{e}_{R}^{2}(n)\right]=E\left[\bar{e}_{I}^{2}(n)\right]=\sigma_{e}^{2}$, and $E[\cdot]$ denotes the expectation operator.

To remove the channel distortion, a $T_{\mathrm{s}} / 2$-spaced equalizer is employed, which is defined by

$$
\bar{y}(n)=\sum_{i=0}^{2 m-1} \bar{w}_{i} \bar{r}(n-i)=\overline{\mathbf{w}}^{\mathrm{T}} \overline{\mathbf{r}}(n)
$$

where $2 m$ is the order or length of the $T_{\mathrm{s}} / 2$-spaced equalizer,

$$
\overline{\mathbf{w}}=\left[\begin{array}{llll}
\bar{w}_{0} & \bar{w}_{1} & \cdots & \bar{w}_{2 m-1}
\end{array}\right]^{\mathrm{T}}
$$

is the equalizer complex-valued weight vector, and

$$
\overline{\mathbf{r}}(n)=\left[\begin{array}{llll}
\bar{r}(n) & \bar{r}(n-1) & \cdots & \bar{r}(n-2 m+1)
\end{array}\right]^{\mathrm{T}}
$$

is the equalizer input vector. To deal with non-minimum phase channels, the equalizer should have a decision delay approximately to $m$. Before blind adaptation, the equalizer weights are initialized to $\bar{w}_{i}=1+j 0$ for $i=m-1$ and $m$, and $\bar{w}_{i}=0+j 0$ for all the other values of $i$. The FSE output $\bar{y}(n)$ is decimated by a factor of 2 to create the $T_{\mathrm{s}}$-spaced output $y(k)$.

It can easily be shown [17] that the system model of Figure 1 is equivalent to the model depicted in Figure 2 by defining

$$
\begin{aligned}
& \overline{\mathbf{a}}^{\mathrm{e}}=\left[\begin{array}{llll}
\bar{a}_{0} & \bar{a}_{2} & \cdots & \bar{a}_{2 N_{\mathrm{c}}-2}
\end{array}\right]^{\mathrm{T}}, \quad \overline{\mathbf{a}}^{\mathrm{o}}=\left[\begin{array}{llll}
\bar{a}_{1} & \bar{a}_{3} & \cdots & \bar{a}_{2 N_{\mathrm{c}}-1}
\end{array}\right]^{\mathrm{T}} \\
& \overline{\mathbf{w}}^{\mathrm{e}}=\left[\begin{array}{llll}
\bar{w}_{0} & \bar{w}_{2} & \cdots & \bar{w}_{2 m-2}
\end{array}\right]^{\mathrm{T}}, \quad \overline{\mathbf{w}}^{\mathrm{O}}=\left[\begin{array}{llll}
\bar{w}_{1} & \bar{w}_{3} & \cdots & \bar{w}_{2 m-1}
\end{array}\right]^{\mathrm{T}}
\end{aligned}
$$

and

$$
\begin{array}{ll}
e^{\mathrm{e}}(k)=\bar{e}(2 n), & e^{\mathrm{o}}(k)=\bar{e}(2 n+1) \\
r^{\mathrm{e}}(k)=\bar{r}(2 n), & r^{\mathrm{o}}(k)=\bar{r}(2 n+1)
\end{array}
$$




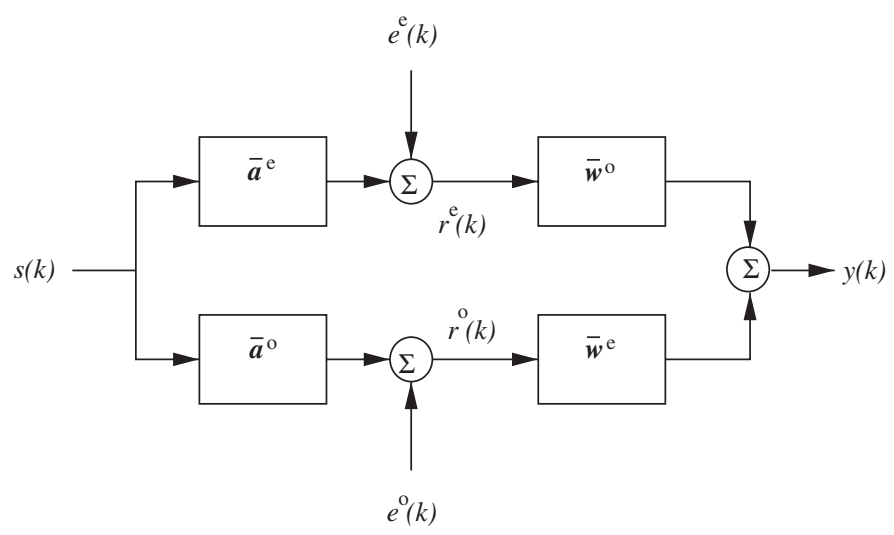

Figure 2. Multichannel model of communication system with $T_{\mathrm{s}} / 2$-spaced equalizer, where $T_{\mathrm{s}}$ denotes symbol period, and the index $k$ indicates $T_{\mathrm{s}}$-spaced quantities.

Further define

$$
\mathbf{w}=\left[\begin{array}{llll}
w_{0} & w_{1} & \cdots & w_{2 m-1}
\end{array}\right]^{\mathrm{T}}=\left[\begin{array}{ll}
\left(\overline{\mathbf{w}}^{\mathrm{o}}\right)^{\mathrm{T}} & \left(\overline{\mathbf{w}}^{\mathrm{e}}\right)^{\mathrm{T}}
\end{array}\right]^{\mathrm{T}}
$$

and

$$
\mathbf{r}(k)=\left[\begin{array}{llll}
r(k) & r(k-1) & \cdots & r(k-2 m+1)
\end{array}\right]^{\mathrm{T}}=\left[\left(\mathbf{r}^{\mathrm{e}}(k)\right)^{\mathrm{T}}\left(\mathbf{r}^{\mathrm{O}}(k)\right)^{\mathrm{T}}\right]^{\mathrm{T}}
$$

with $\mathbf{r}^{\mathrm{e}}(k)=\left[\begin{array}{lllll}r^{\mathrm{e}}(k) & r^{\mathrm{e}}(k-1) & \cdots & r^{\mathrm{e}}(k-m+1)\end{array}\right]^{\mathrm{T}}$ and $\mathbf{r}^{\mathrm{o}}(k)=\left[\begin{array}{llll}r^{\mathrm{o}}(k) & r^{\mathrm{o}}(k-1) & \cdots & r^{\mathrm{o}}(k-m+1)\end{array}\right]^{\mathrm{T}}$ Then the $T_{\mathrm{s}}$-spaced equalizer output $y(k)$ is given by

$$
y(k)=\sum_{i=0}^{2 m-1} w_{i} r(k-i)=\mathbf{w}^{\mathrm{T}} \mathbf{r}(k)
$$

The equalizer model (12) forms the basis for the discussion of the blind adaptive algorithms in the following subsections.

\subsection{CMA-based equalizer}

The CMA adjusts the equalizer weights by minimizing the non-convex cost function

$$
\bar{J}_{\mathrm{CMA}}(\mathbf{w})=E\left[\left(|y(k)|^{2}-\Delta_{2}\right)^{2}\right]
$$

using a stochastic gradient algorithm, where $\Delta_{2}$ is a real positive constant defined by

$$
\Delta_{2}=E\left[|s(k)|^{4}\right] / E\left[|s(k)|^{2}\right]
$$

At $T_{\mathrm{s}}$-spaced sample $k$, given $y(k)=\mathbf{w}^{\mathrm{T}}(k) \mathbf{r}(k)$, the CMA adapts $\mathbf{w}$ according to [2,3]

$$
\begin{aligned}
\varepsilon(k) & =y(k)\left(\Delta_{2}-|y(k)|^{2}\right) \\
\mathbf{w}(k+1) & =\mathbf{w}(k)+\mu \varepsilon(k) \mathbf{r}^{*}(k)
\end{aligned}
$$

where $\mu$ is a small positive adaptive gain and $\mathbf{r}^{*}(k)$ is the complex conjugate of $\mathbf{r}(k)$. Typically, a very small adaptive gain $\mu$ has to be used to ensure convergence.

The CMA has a very simple computational complexity, as is summarized in Table I, and it is by far the most popular blind equalizer for high-order QAM signal constellation. Although 
Table I. Comparison of computational complexity per weight update. The equalizer order is $2 m$.

\begin{tabular}{lccc}
\hline Equalizer & Multiplications & Additions & $\exp (\cdot)$ \\
\hline CMA & $8 \times 2 m+6$ & $8 \times 2 m$ & - \\
CMA + DD & $16 \times 2 m+8$ & $20 \times 2 m$ & - \\
CMA + SDD & $12 \times 2 m+29$ & $14 \times 2 m+21$ & 4 \\
\hline
\end{tabular}

$M$-QAM symbols do not fall on the circle of radius $\sqrt{\Delta_{2}}$, it is known that the cost function $\bar{J}_{\mathrm{CMA}}(\mathbf{w})$ is minimized at the equalizer weight solution which restores the signal constellation. Under certain conditions, the CMA converges to this solution subject to a possible phase shift. The CMA is also known to be very robust and is capable of opening 'initially closed eye'.

\subsection{Concurrent CMA and decision-directed equalizer}

De Castro et al. [7] proposed a blind equalization scheme that consists of a CMA equalizer and a DD equalizer operating concurrently. Specifically, let

$$
\mathbf{w}=\mathbf{w}_{c}+\mathbf{w}_{d}
$$

Here $\mathbf{w}_{c}$ is the weight vector of the CMA equalizer which is designed to minimize the CMA cost function $\bar{J}_{\mathrm{CMA}}(\mathbf{w})$ by adapting $\mathbf{w}_{c}$, while $\mathbf{w}_{d}$ is the weight vector of the DD equalizer which is designed to minimize the decision-based MSE

$$
\bar{J}_{\mathrm{DD}}(\mathbf{w})=\frac{1}{2} E\left[|\mathscr{Q}[y(k)]-y(k)|^{2}\right]
$$

by adjusting $\mathbf{w}_{d}$, where $2[y(k)]$ denotes the quantized equalizer output defined by

$$
\mathscr{2}[y(k)]=\arg \min _{s_{i l} \in \mathscr{S}}\left|y(k)-s_{i l}\right|^{2}
$$

More precisely, at symbol-spaced sample $k$, given

$$
y(k)=\mathbf{w}_{c}^{\mathrm{T}}(k) \mathbf{r}(k)+\mathbf{w}_{d}^{\mathrm{T}}(k) \mathbf{r}(k)
$$

the CMA part adapts $\mathbf{w}_{c}$ according to rule (15) by substituting $\mathbf{w}_{c}$ in the place of $\mathbf{w}$ with an adaptive gain $\mu_{c}$. The DD adaptation follows immediately after the CMA adaptation but it only takes place if the CMA adjustment is viewed to be a successful one. Let

$$
\tilde{y}(k)=\mathbf{w}_{c}^{\mathrm{T}}(k+1) \mathbf{r}(k)+\mathbf{w}_{d}^{\mathrm{T}}(k) \mathbf{r}(k)
$$

Then the DD part adjusts $\mathbf{w}_{d}$ according to [7]

$$
\mathbf{w}_{d}(k+1)=\mathbf{w}_{d}(k)+\mu_{d} \delta(2[\tilde{y}(k)]-2[y(k)])(2[y(k)]-y(k)) \mathbf{r}^{*}(k)
$$

where $\mu_{d}$ is the adaptive gain of the DD equalizer and the indicator function

$$
\delta(x)= \begin{cases}1, & x=0+j 0 \\ 0, & x \neq 0+j 0\end{cases}
$$

It can be seen that $\mathbf{w}_{d}$ is updated only if the equalizer hard decisions before and after the CMA adaptation are the same. A potential problem of (hard) DD adaptation is that if the decision is wrong, error propagation occurs which subsequently degrades equalizer adaptation. As analysed in [7], if the equalizer hard decisions before and after the CMA adaptation are the same, the decision probably is a right one. The DD adaptation, when is safe to perform, has a 
much faster convergence speed and is capable of lowering the steady-state MSE, compared with the pure CMA. The complexity of this concurrent CMA + DD blind equalizer, summarized in Table I, is still linear in the equalizer order $2 \mathrm{~m}$. Obviously, this CMA + DD blind equalizer combines the advantages of both the CMA and DD adaptation. It does not suffer from a serious phase shift problem as the CMA does, and automatically performs a gain control or scaling for the restoration of the signal constellation (1). The adaptive gain $\mu_{d}$ for the DD equalizer can often be chosen much larger than $\mu_{c}$ for the CMA. Nevertheless, care must be exercised in choosing $\mu_{d}$ to avoid setting it to a too large value, which would cause serious error propagation due to incorrect decisions.

\subsection{Concurrent CMA and soft decision-directed equalizer}

After the equalization is accomplished, the equalizer soft output $y(k)$ can approximately be expressed in two terms:

$$
y(k) \approx x(k)+v(k)
$$

where $x(k)=s\left(k-k_{d}\right), k_{d}$ is an integer, and $v(k)=v_{R}(k)+j v_{I}(k)$ is approximately a Gaussian white noise. Thus, if the equalizer weights have correctly been chosen, the equalizer output can be modelled approximately by $M$ Gaussian clusters with the cluster means being $s_{i l}$ for $1 \leqslant i$, $l \leqslant Q$. All the clusters have an approximate covariance

$$
\left[\begin{array}{cc}
E\left[v_{R}^{2}(k)\right] & E\left[v_{R}(k) v_{I}(k)\right] \\
E\left[v_{I}(k) v_{R}(k)\right] & E\left[v_{I}^{2}(k)\right]
\end{array}\right] \approx\left[\begin{array}{cc}
\rho & 0 \\
0 & \rho
\end{array}\right]
$$

Under the above conditions, the a posteriori probability density function (p.d.f.) of $y(k)$ is approximately

$$
p(\mathbf{w}, y(k)) \approx \sum_{q=1}^{Q} \sum_{l=1}^{Q} \frac{p_{q l}}{2 \pi \rho} \exp \left(-\frac{\left|y(k)-s_{q l}\right|^{2}}{2 \rho}\right)
$$

where $p_{q l}$ are the a priori probabilities of $s_{q l}, 1 \leqslant q, l \leqslant Q$, and they are all equal.

The computation of the p.d.f. (25) involves the evaluation of $M \exp (\cdot)$ function values. A local approximation can be adopted for this p.d.f. which only evaluates four $\exp (\cdot)$ function values. This is achieved by dividing the complex plane into $M / 4$ regular regions, as illustrated in Figure 3. Each region $S_{i, l}$ contains four symbol points

$$
S_{i, l}=\left\{s_{p q}, p=2 i-1,2 i, q=2 l-1,2 l\right\}
$$

If the equalizer output $y(k)$ is within the region $S_{i, l}$, a local approximation to the a posteriori p.d.f. of $y(k)$ is

$$
\hat{p}(\mathbf{w}, y(k)) \approx \sum_{p=2 i-1}^{2 i} \sum_{q=2 l-1}^{2 l} \frac{1}{8 \pi \rho} \exp \left(-\frac{\left|y(k)-s_{p q}\right|^{2}}{2 \rho}\right)
$$

where each a priori probability has been set to $\frac{1}{4}$. Obviously this approximation is only valid when the equalization goal has been accomplished. A bootstrap optimization process, however, can be performed to achieve the MAP solution, as is presented in References $[12,13]$.

The proposed scheme operates a CMA equalizer and a SDD equalizer concurrently. The CMA part is identical to that of the concurrent CMA + DD scheme. The purpose of this CMA sub-equalizer is to open the eye, so that the local p.d.f. expression (27) is approximately valid. 


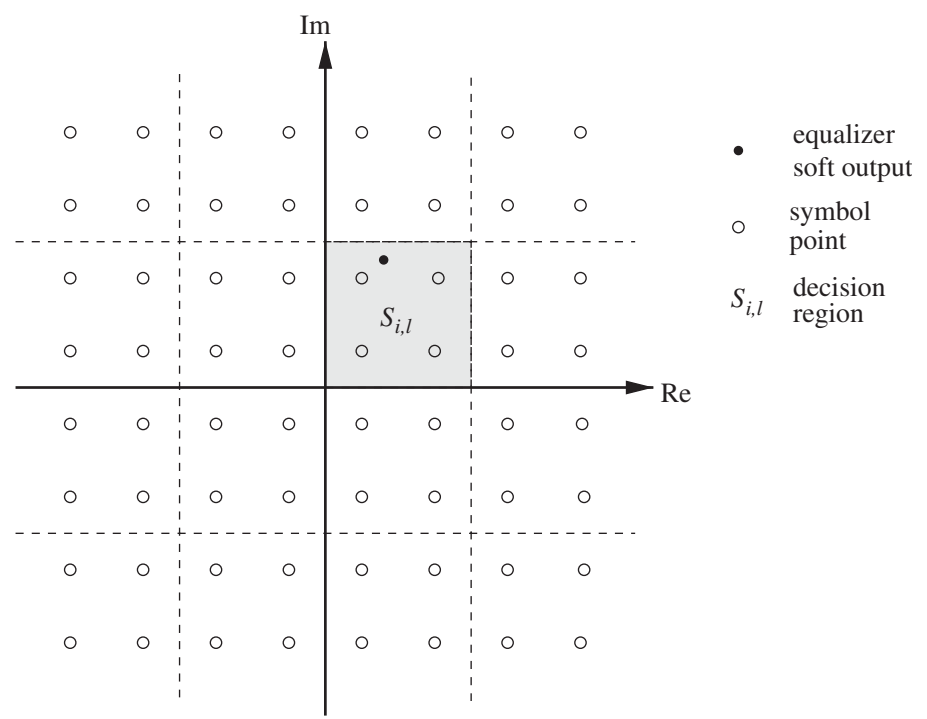

Figure 3. Illustration of local decision regions for soft decision-directed adaptation with 64-QAM constellation.

The SDD equalizer is designed to maximize $\log$ of the local a posteriori p.d.f. criterion

$$
\bar{J}_{\text {LMAP }}(\mathbf{w})=E\left[J_{\text {LMAP }}(\mathbf{w}, y(k))\right]
$$

by adjusting $\mathbf{w}_{d}$ using a stochastic gradient algorithm, where

$$
J_{\text {LMAP }}(\mathbf{w}, y(k))=\rho \log (\hat{p}(\mathbf{w}, y(k)))
$$

Specifically, the SDD equalizer adapts $\mathbf{w}_{d}$ according to

$$
\mathbf{w}_{d}(k+1)=\mathbf{w}_{d}(k)+\mu_{d} \frac{\partial J_{\mathrm{LMAP}}(\mathbf{w}(k), y(k))}{\partial \mathbf{w}_{d}}
$$

where

$$
\frac{\partial J_{\text {LMAP }}(\mathbf{w}, y(k))}{\partial \mathbf{w}_{d}}=\frac{\sum_{p=2 i-1}^{2 i} \sum_{q=2 l-1}^{2 l} \exp \left(-\left(\left|y(k)-s_{p q}\right|^{2}\right) / 2 \rho\right)\left(s_{p q}-y(k)\right)}{\sum_{p=2 i-1}^{2 i} \sum_{q=2 l-1}^{2 l} \exp \left(-\left(\left|y(k)-s_{p q}\right|^{2}\right) / 2 \rho\right)} \mathbf{r}^{*}(k)
$$

and $\mu_{d}$ is an adaptive gain. The choice of $\rho$ should ensure a proper separation of the four clusters in $S_{i, l}$. If the value of $\rho$ is too large, a desired degree of separation may not be achieved. On the other hand, if a too small $\rho$ is used, the algorithm attempts to impose a very tight control in the size of clusters and may fail to do so. Apart from these two extreme cases, the performance of the algorithm does not critically depend on the value of $\rho$. As the minimum distance between the two neighbouring symbol points is 2 , typically $\rho$ is chosen to be less than 1 .

Soft decision nature is evident in (31). Rather than committed to a single hard decision $2[y(k)]$ as the DD scheme does, alternative decisions are also considered in a local region $S_{i, l}$ that includes $2[y(k)]$, and each tentative decision is weighted by an exponential term $\exp (\cdot)$ which is a function of the distance between the equalizer soft output $y(k)$ and the tentative decision $s_{p q}$. This soft decision nature enables a simultaneous update of $\mathbf{w}_{c}$ and $\mathbf{w}_{d}$ without worrying about 
error propagation and, therefore, simplifies the operation. It also has an effect that a larger adaptive gain $\mu_{d}$ can often be used, compared with the DD scheme. It is also obvious that this SDD scheme corresponds to the last stage of the bootstrap MAP scheme given in References $[12,13]$. The complexity of this CMA + SDD scheme is given in Table I, where it can be seen that computational complexity per weight update of this proposed new scheme is simpler than that of the CMA + DD scheme. The four $\exp (\cdot)$ evaluations can be implemented through look up table in practice.

\section{SIMULATION STUDY}

The performance of the CMA + SDD and CMA + DD blind equalizers were evaluated in a computer simulation using the standard CMA blind equalizer as a benchmark. For the case of constant channels, two performance criteria were used to assess the convergence rate of a blind equalizer. The first one was a decision-based estimated MSE at each adaptation sample based on a block of $N_{\text {MSE }} T_{\mathrm{s}}$-spaced data samples

$$
\mathrm{MSE}=\frac{1}{N_{\mathrm{MSE}}} \sum_{k=1}^{N_{\mathrm{MSE}}}|2[y(k)]-y(k)|^{2}
$$

The second one was the maximum distortion (MD) measure defined by

$$
\mathrm{MD}=\frac{\sum_{i=0}^{N_{f}-1}\left|f_{i}\right|-\left|f_{i_{\max }}\right|}{\left|f_{i_{\max }}\right|}
$$

where $\left\{f_{i}\right\}_{i=0}^{N_{f}-1}$ was the combined impulse response of the channel and equalizer defined by $\overline{\mathbf{w}}^{\mathrm{o}} \star \overline{\mathbf{a}}^{\mathrm{e}}+\overline{\mathbf{w}}^{\mathrm{e}} \star \overline{\mathbf{a}}^{\mathrm{o}}$ with $\star$ denoting convolution and $N_{f}=N_{c}+m-1$ being the length of the $T_{\mathrm{s}}$-spaced combined impulse response, and

$$
f_{i_{\max }}=\max \left\{f_{i}, 0 \leqslant i \leqslant N_{f}-1\right\}
$$

The equalizer output signal constellation after convergence was also shown using $N_{\text {test }}=6000 T_{\mathrm{s}}$-spaced testing data samples not used in adaptation.

The $T_{\mathrm{s}} / 2$-spaced equalizer order $2 m$ should be chosen sufficiently long to ensure the capability of opening closed eye and good steady-state performance, but not too long which can cause the problems of seriously enhancing noise and slow convergence rate. In the simulation study, the actual value of $2 m$ used was found empirically. The values of various adaptive algorithm parameters, namely adaptive gains for the CMA, DD and SDD, and the cluster width for the SDD, were also optimized empirically to ensure fast convergence speed and good steady-state performance. Extensive simulation had been performed, but only two sets of results are presented here due to the space limitation.

\section{Example 1}

This was a time-invariant system. In this example, 256-QAM data symbols were transmitted through a $T_{\mathrm{s}} / 2$-spaced 22-tap channel whose CIR is given in Table II. The noise power was

set to $\sigma_{e}^{2}=4.24 \times 10^{-5}$, corresponding to a channel signal to noise ratio (SNR) of $60 \mathrm{~dB}$. The $T_{\mathrm{s}} / 2$-spaced equalizer had 26 taps and the length of the data block for estimating the MSE at each adaptation was $N_{\mathrm{MSE}}=1000$. The adaptive gain for the CMA had to be set to $\mu_{c}=10^{-8}$ to avoid divergence. The two adaptive gains of the CMA +DD equalizer were set to $\mu_{c}=10^{-8}$ and 
Table II. A simulated $T_{\mathrm{S}} / 2$-spaced 22-tap channel impulse response, where $T_{\mathrm{S}}$ denotes symbol period.

\begin{tabular}{ccrcrr}
\hline Tap No. & \multicolumn{1}{c}{$\operatorname{Re}$} & $\mathrm{Im}$ & Tap No. & \multicolumn{1}{c}{$\operatorname{Re}$} & \multicolumn{1}{c}{$\mathrm{Im}$} \\
\hline 0 & 0.0145 & -0.0006 & 11 & 0.0294 & -0.0049 \\
1 & 0.0750 & 0.0176 & 12 & -0.0181 & 0.0032 \\
2 & 0.3951 & 0.0033 & 13 & 0.0091 & 0.0003 \\
3 & 0.7491 & -0.1718 & 14 & -0.0038 & -0.0023 \\
4 & 0.1951 & 0.0972 & 15 & 0.0019 & 0.0027 \\
5 & -0.2856 & 0.1896 & 16 & -0.0018 & -0.0014 \\
6 & 0.0575 & -0.2096 & 17 & 0.0006 & 0.0003 \\
7 & 0.0655 & 0.1139 & 18 & 0.0005 & 0.0000 \\
8 & -0.0825 & -0.0424 & 19 & -0.0008 & -0.0001 \\
9 & 0.0623 & 0.0085 & 20 & 0.0000 & -0.0002 \\
10 & -0.0438 & 0.0034 & 21 & 0.0001 & 0.0006 \\
\hline
\end{tabular}
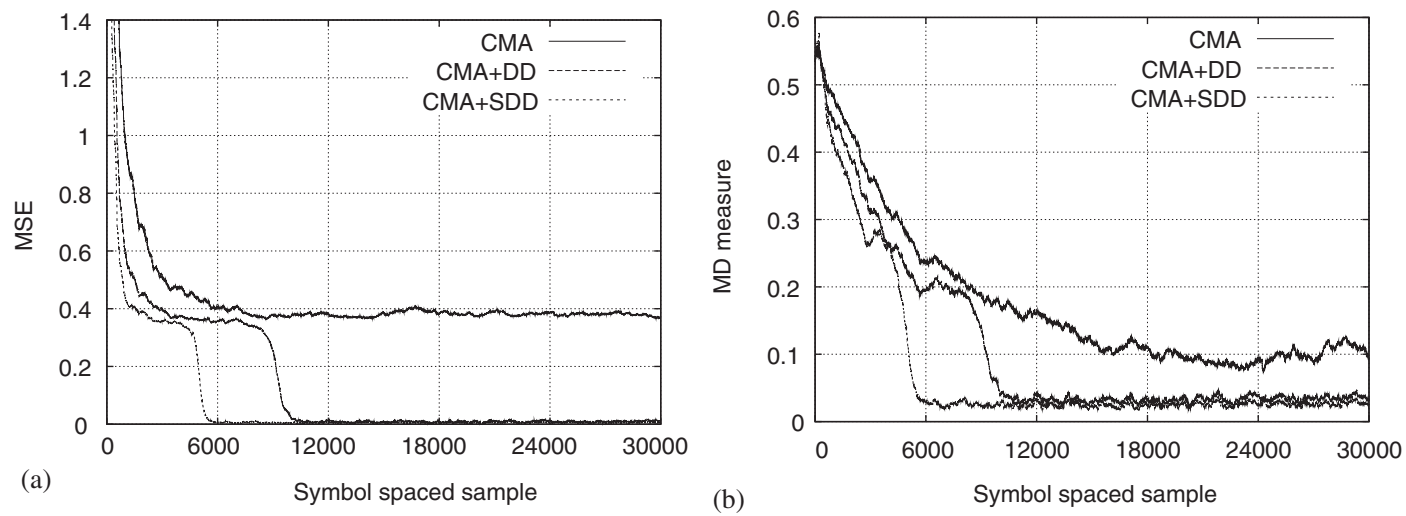

Figure 4. Comparison of convergence performance in terms of (a) estimated MSE; and (b) MD measure for the time-invariant channel.

$\mu_{d}=10^{-5}$. For the CMA + SDD equalizer, the two adaptive gains were set to $\mu_{c}=10^{-8}$ and $\mu_{d}=2 \times 10^{-5}$ with a width $\rho=0.4$. Note that these values of adaptive gains were found to be optimal empirically.

The learning curves of the three blind equalizers, in terms of the estimated MSE and MD measure, are depicted in Figure 4(a) and 4(b), respectively, while the equalizer output signal constellations after convergence are illustrated in Figure 5. For this example, faster convergence speed of the proposed new scheme over the CMA + DD scheme can clearly be seen. The results also indicate that the steady-state equalization performance of the CMA + SDD algorithm is slightly better than the CMA + DD algorithm.

\section{Example 2}

This example was a 64-QAM fading channel operated at 4 Msymbols/s. The baseband continuous-time system was simulated. The transmission pulse had a raised-cosine characteristics with a rolloff factor 0.5 and was split equally between the transmitter and receiver filters. 

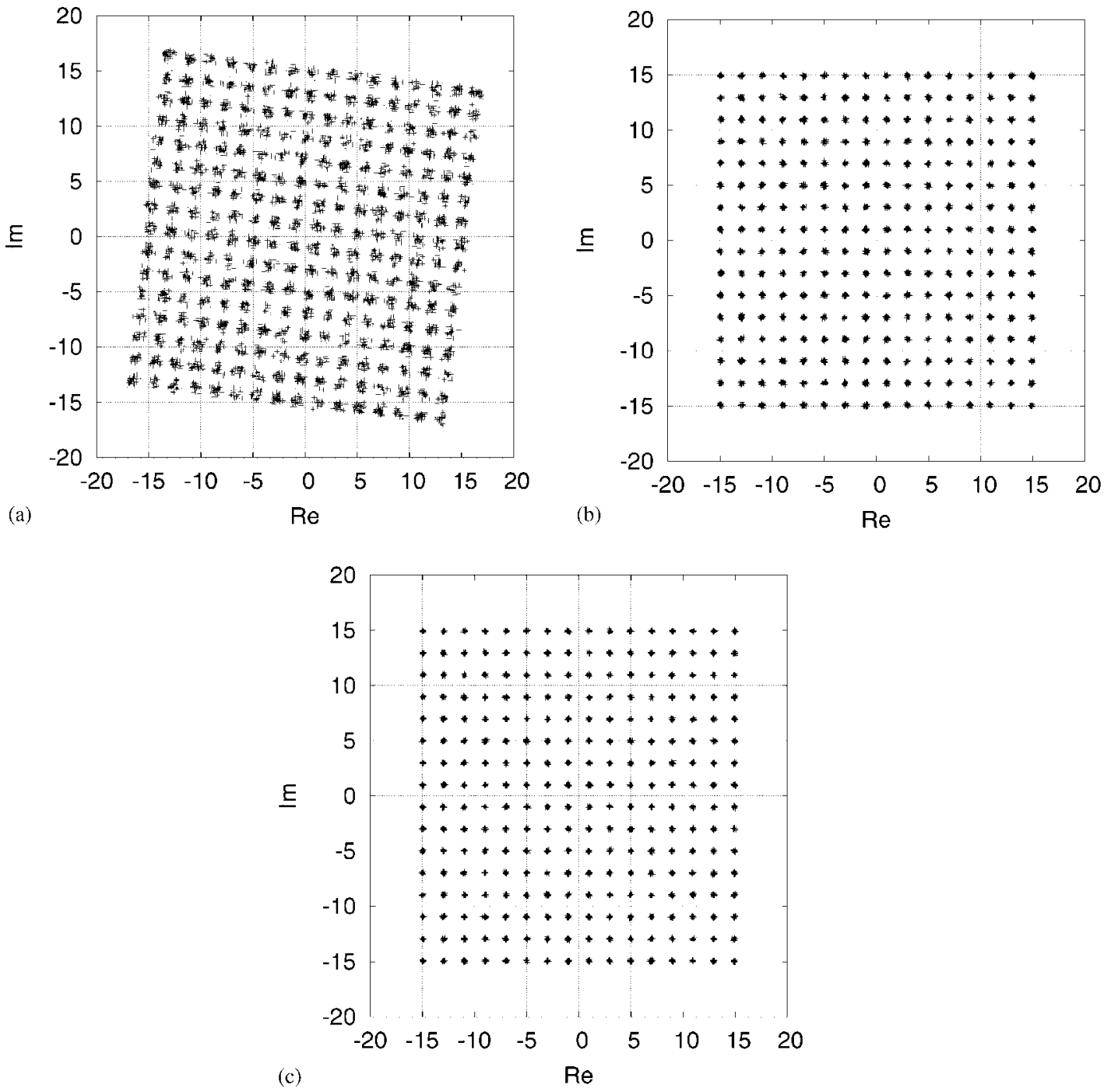

Figure 5. Equalizer output signal constellations after convergence: (a) the CMA; (b) the CMA+DD; and (c) CMA + SDD for the time-invariant channel.

The fading channel was implemented using the following tap-delay line model

$$
v(t)=c_{0}(t) u(t)+c_{1}(t) u\left(t-\tau_{1}\right)+c_{2}(t) u\left(t-\tau_{2}\right)
$$

where $u(t)$ was the transmitter output and $v(t)$ was the fading channel output; $\tau_{1} \approx 0.44 T_{\mathrm{s}}$ and $\tau_{2} \approx 1.13 T_{\mathrm{s}}$; the magnitudes of the complex-valued tap weights $c_{i}(t), 0 \leqslant i \leqslant 2$, were i.i.d. Rayleigh processes and the root mean powers of the both real and imaginary components of $c_{i}(t)$ were [0.7 0.60 .5 , for $0 \leqslant i \leqslant 2$, respectively. Fading was continuously at a fading rate of $10 \mathrm{~Hz}$. The noise powers was $\sigma_{e}^{2}=0.011$. Receiver outputs were sampled at twice of the symbol rate and passed to an equalizer of $8 T_{\mathrm{s}} / 2$-spaced taps. 

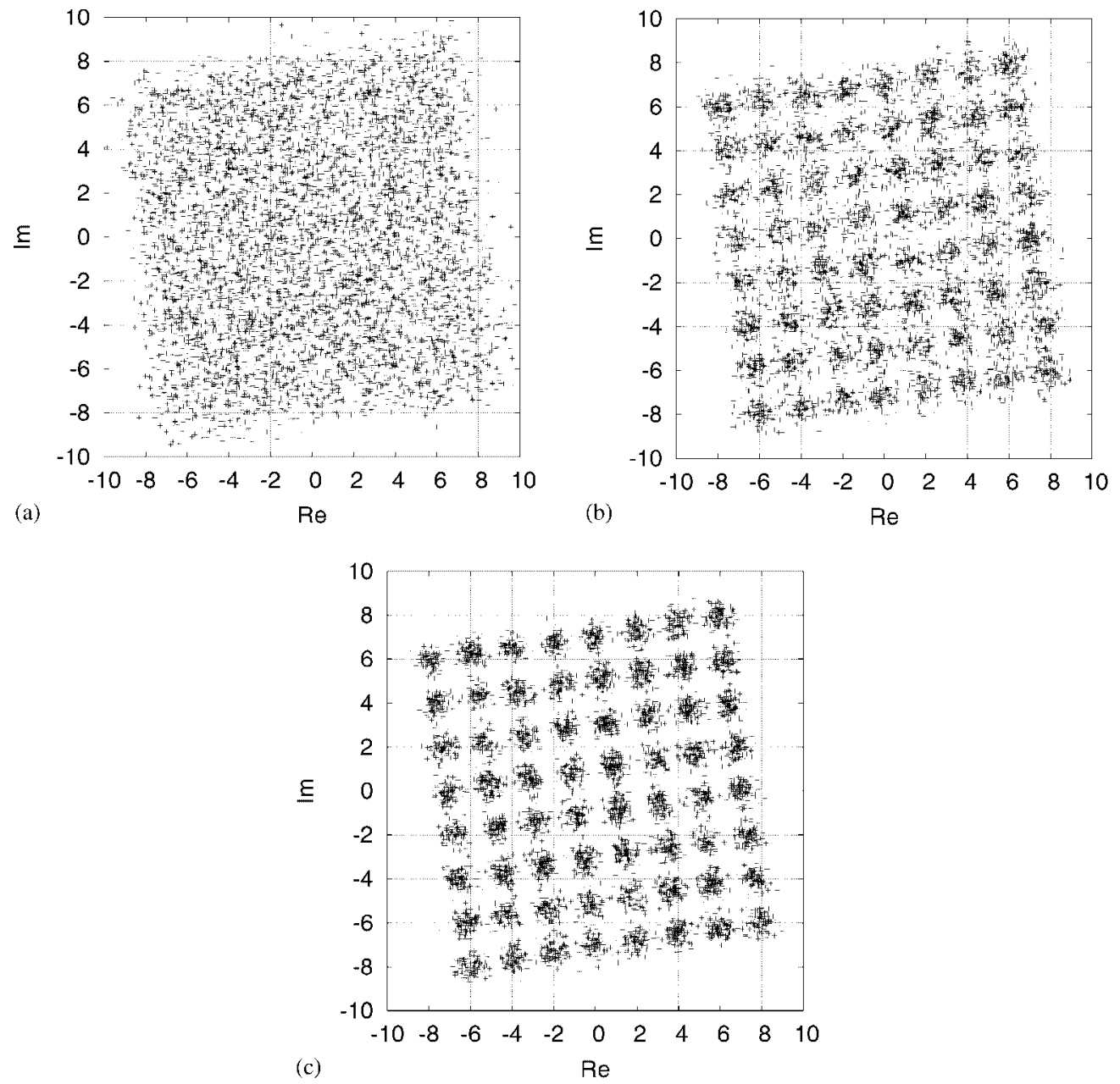

Figure 6. CMA equalizer output signal constellations for the fading channel: (a) after adaptation of 20000 symbols; (b) after adaptation of 25000 symbols; and (c) after adaptation of 30000 symbols. Six thousand $T_{\mathrm{s}}$-spaced samples were used in showing the signal constellation with continuous adaptation.

An appropriate value of the adaptive gain for the CMA equalizer was found to be $\mu_{c}=10^{-6}$. The two adaptive gains of the concurrent CMA +DD equalizers were set to $\mu_{c}=10^{-6}$ and $\mu_{d}=0.001$. For the CMA + SDD equalizer, the two adaptive gains were chosen as $\mu_{c}=10^{-6}$ and $\mu_{d}=0.002$, and the cluster width was set to $\rho=0.4$. Figure 6 depicts the CMA equalizer output signal constellations after (a) adaptation of 20000 symbols, (b) adaptation of 25000 symbols, and (c) adaptation of 30000 symbols. Each signal constellation was shown with 6000 $T_{\mathrm{s}}$-spaced samples with continuous adaptation, as the channel was time-varying. Similarly, the convergence performance of the CMA + DD and CMA + SDD blind equalizers are demonstrated in Figures 7 and 8, respectively. The results clearly show that the CMA + SDD scheme had the fastest convergence rate. 

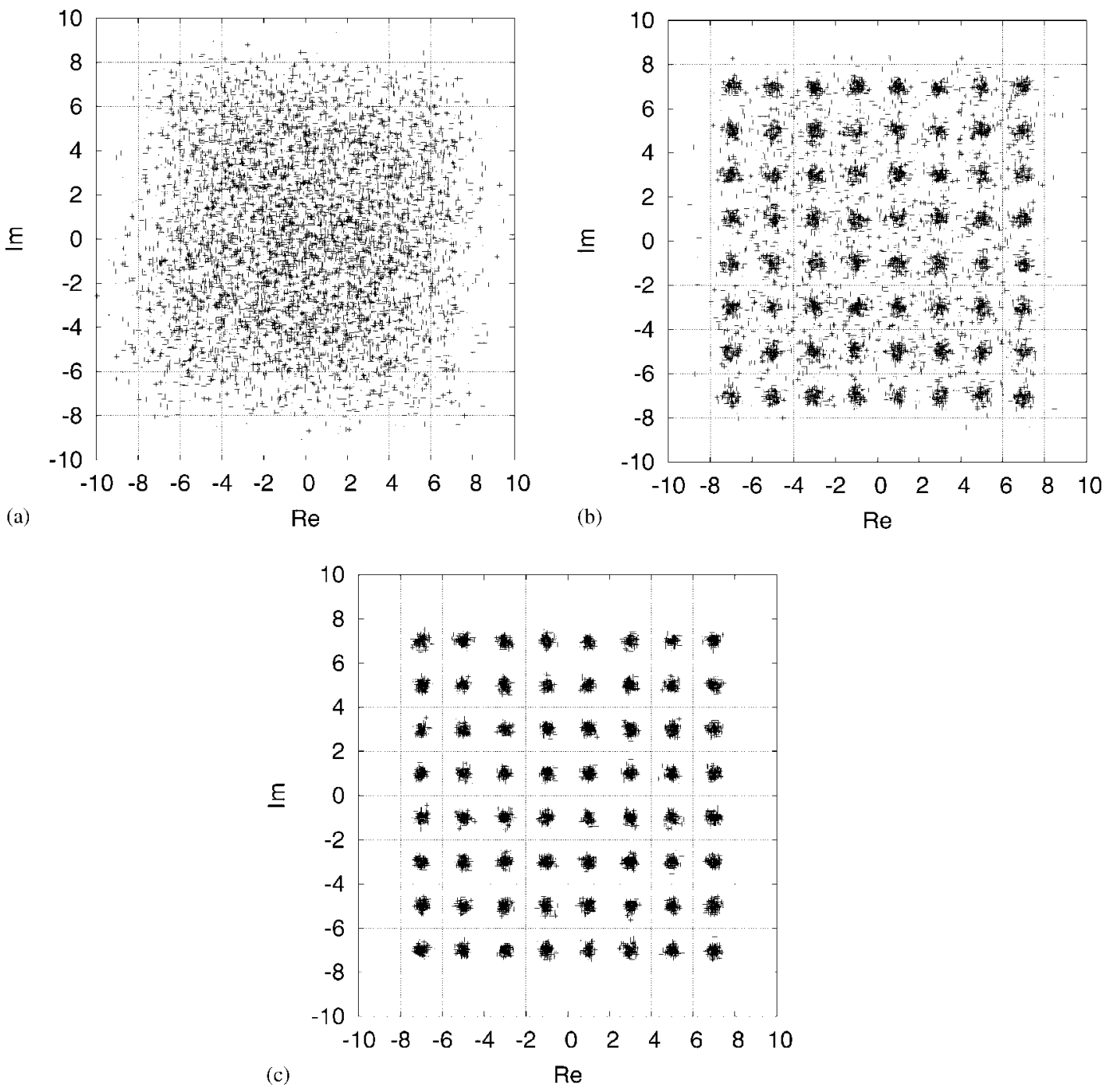

Figure 7. CMA + DD equalizer output signal constellations for the fading channel: (a) after adaptation of 15000 symbols; (b) after adaptation of 20000 symbols; and (c) after adaptation of 25000 symbols. Six thousand $T_{\mathrm{s}}$-spaced samples were used in showing the signal constellation with continuous adaptation.

\section{CONCLUSIONS}

In this paper, a novel low-complexity fractionally spaced blind equalization scheme has been proposed based on operating a CMA equalizer and a SDD equalizer concurrently. Compared with a state-of-art low-complexity blind equalization scheme, namely the recently introduced concurrent CMA and DD blind equalizer, the proposed concurrent CMA and SDD blind equalizer has simpler computational requirements, faster convergence rate and slightly better steady-state equalization performance. This new blind equalizer, together with the concurrent 

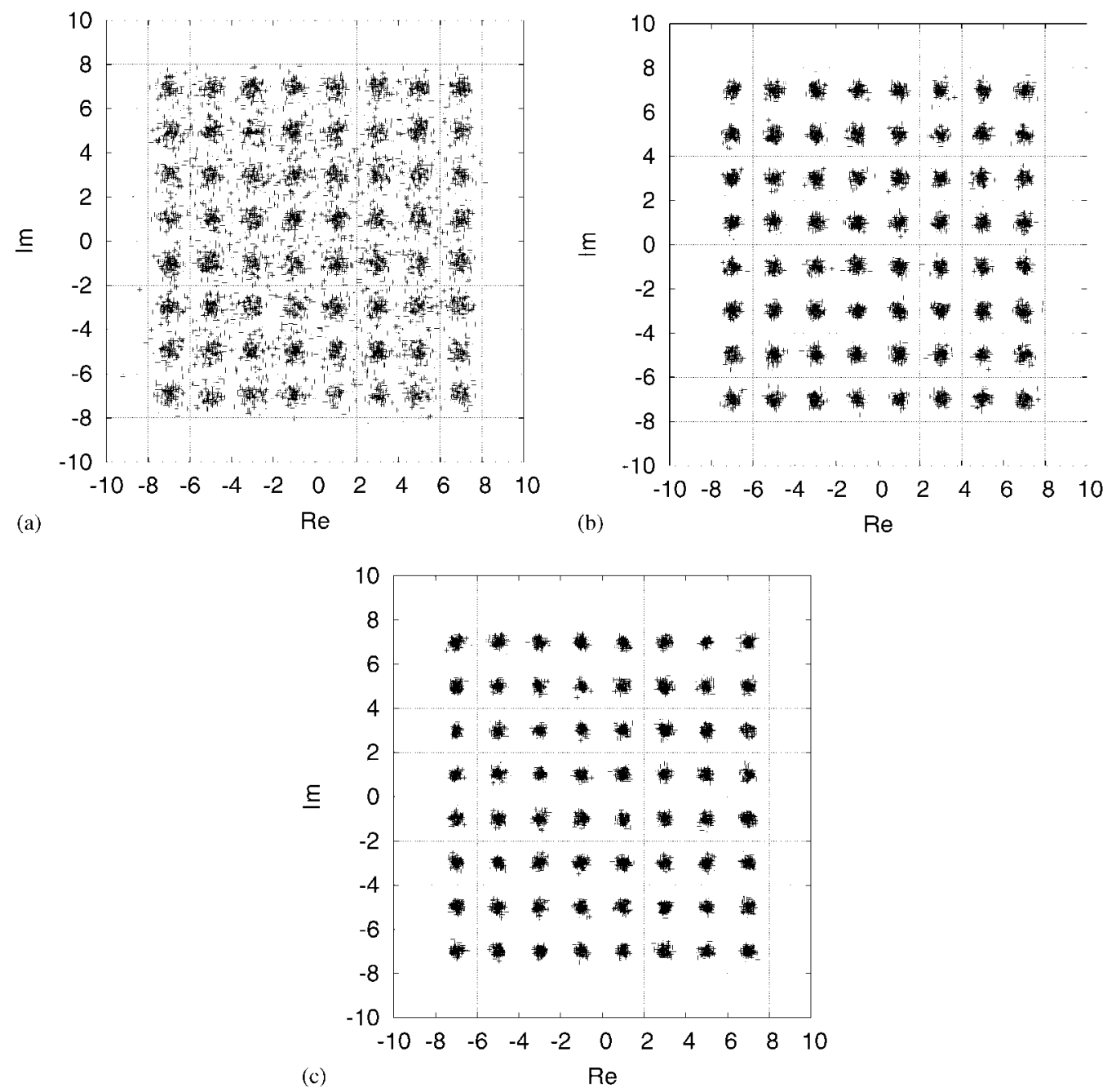

Figure 8. CMA + SDD equalizer output signal constellations for the fading channel: (a) after adaptation of 15000 symbols; (b) after adaptation of 20000 symbols; and (c) after adaptation of 25000 symbols. Six thousand $T_{\mathrm{s}}$-spaced samples were used in showing the signal constellation with continuous adaptation.

CMA and DD blind equalizer, offer practical alternatives to blind equalization of higher-order QAM channels and provide significant equalization improvement over the standard CMAbased blind equalizer.

\section{REFERENCES}

1. Proakis JG. Digital Communications (3rd edn). McGraw-Hill: New York, 1995.

2. Godard D. Self-recovering equalization and carrier tracking in two-dimensional data communication systems. IEEE Transactions on Communications 1980; COM-28:1867-1875. 
3. Treichler JR, Agee BG. A new approach to multipath correction of constant modulus signals. IEEE Transactions on Acoustics Speech and Signal Processing 1983; ASSP-31(2):459-472.

4. Treichler JR. Application of blind equalization techniques to voiceband and RF modems. Preprints of 4th IFAC International Symposium on Adaptive Systems in Control and Signal Processing, France, 1992; 705-713.

5. Jablon NK. Joint blind equalization, carrier recovery, and timing recovery for high-order QAM signal constellations. IEEE Transactions on Signal Processing 1992; 40(6):1383-1398.

6. Macchi O, Eweda E. Convergence analysis of self-adaptive equalizers. IEEE Transactions on Information Theory 1984; IT-3(2):161-176.

7. De Castro FCC, De Castro MCF, Arantes DS. Concurrent blind deconvolution for channel equalization. Proceedings of ICC'2001, vol. 2, Helsinki, Finland, June 11-15, 2001:366-371.

8. Benveniste A, Goursat M. Blind equalizers. IEEE Transactions on Communications 1984; COM-32:871-883.

9. Bellini S, Rocca F. Blind deconvolution: polyspectra or Bussgang techniques? In Digital Communications, Biglieri E, Prati G (eds). North-Holland: Amsterdam, 1986; 251-263.

10. Picchi G, Prati G. Blind equalization and carrier recovering using a 'stop-and-go' decision directed algorithm. IEEE Transactions on Communications 1987; COM-35:877-887.

11. Haykin S (ed.). Blind Deconvolution. Prentice-Hall: Englewood Cliffs, NJ, 1994.

12. Chen S, McLaughlin S, Grant PM, Mulgrew B. Reduced-complexity multi-stage blind clustering equaliser. Proceedings of ICC'1993, vol. 2, Geneva, Switzerland, 1993; 1149-1153.

13. Chen S, McLaughlin S, Grant PM, Mulgrew B. Multi-stage blind clustering equaliser. IEEE Transactions on Communications 1995; 43(3):701-705.

14. Karaoguz J, Ardalan SH. A soft decision-directed blind equalization algorithm applied to equalization of mobile communication channels. Proceedings of ICC'1992, vol. 3, Chicago, U.S.A., 1992; 343.4.1-343.4.5.

15. Chen S, Cook TB, Anderson LC. Blind FIR equalisation for high-order QAM signalling. Proceedings of the 6th International Conference on Signal Processing, Beijing, China, August 26-30, 2002; 1299-1302.

16. Chen S, Cook TB, Anderson LC. A comparative study of two blind FIR equalizers. Digital Signal Processing 2004; 14(1):18-36.

17. Johnson Jr R, Schniter P, Endres TJ, Behm JD, Brown DR, Casas RA. Blind equalization using the constant modulus criterion: a review. Proceedings of IEEE 1998; 86(10):1927-1950. 